\title{
Experiência no curso - contributo para a qualidade do ensino superior
}

\author{
Maria Elisa Chaleta \\ Universidade de Évora
}

\section{Resumo}

A investigação no âmbito do ensino superior estabeleceu forte relação entre a forma como os estudantes aprendem e a percepção que têm sobre os contextos em que essa aprendizagem ocorre. Neste estudo avalia-se a experiência no curso de estudantes do ensino superior em aspectos como Bom Ensino, Avaliação Apropriada, Carga de Trabalho Apropriada e Competências Genéricas. Participaram 872 alunos de $1^{\circ}$ e $3^{\circ}$ ano de cursos de Licenciatura de Ciências Sociais e de Ciências e Tecnologia de três instituições de ensino superior. Os resultados mostram que, na maioria dos cursos, os estudantes consideram existir bom ensino, que a avaliação não é a mais apropriada, que a carga de trabalho é adequada e que este proporciona o desenvolvimento de competências genéricas. Uma significativa maioria indica estar satisfeita com a qualidade dos cursos.

Palavras-chave: Experiência no curso. Qualidade da aprendizagem e do ensino.Ensino superior.

\section{Course experience - contribution to the quality of higher education}

\section{Abstract}

This research in the field of higher education established strong relationship between how students learn and their perception about the contexts in which this learning takes place. In this study, we aimed to evaluate the experience in the course of higher education students. Evaluates aspects such as Good Teaching, Appropriate Assessment, Appropriate Workload and Generic Skills. 872 students of 1 st and 3rd year undergraduate courses in Social Sciences and Science and Technology of three institutions of higher education attended. The results indicate that, in most courses, students feel that there is good teaching, the assessment is not the most appropriate, the workload is adequate and that provides the development of generic skills. A significant majority indicated of being satisfied with the quality of the courses.

Keywords: Course Experience. Quality of learning and teaching. Higher education. 
Experiência no curso - contributo para a qualidade do ensino superior

\section{Experiencia del curso - contribución a la calidad de la educación superior}

\section{Resumo}

A investigação no âmbito do ensino superior estabeleceu forte relação entre a forma como os estudantes aprendem e a percepção que têm sobre os contextos em que essa aprendizagem ocorre. Neste estudo avalia-se a experiência no curso de estudantes do ensino superior em aspectos como Bom Ensino, Avaliação Apropriada, Carga de Trabalho Apropriada e Competências Genéricas. Participaram 872 alunos de $1^{\circ}$ e $3^{\circ}$ ano de cursos de Licenciatura de Ciências Sociais e de Ciências e Tecnologia de três instituições de ensino superior. Os resultados mostram que, na maioria dos cursos, os estudantes consideram existir bom ensino, que a avaliação não é a mais apropriada, que a carga de trabalho é adequada e que este proporciona o desenvolvimento de competências genéricas. Uma significativa maioria indica estar satisfeita com a qualidade dos cursos.

Palavras-chave: Experiência no curso. Qualidade da aprendizagem e do ensino. Ensino superior.

\section{Introdução}

A implementação do Processo de Bolonha,nos países europeus, encerrava um conjunto de pressupostos que incidiam, particularmente, na mudança de paradigma aonível do processo de ensino/aprendizagem (sendo a tônica colocada na aprendizagem autônoma dos estudantes), a adopção de ECTS (European Credit Transfer System), a oferta de mais unidades curriculares optativas para currículos mais diversificados considerando as necessidades do mercado de trabalho, a redução dos cursos de primeiro ciclo para três anos e de segundo ciclo (mestrado) para dois, o que permitiria a equiparação e comparabilidade da formação em termos europeuse facilitaria a mobilidade de docentes e discentes. Apesar da etaparelativa à reestruturação curricular em Portugal ter sido concluída em 2010 verifica-se, passados quatro anos, que as questões relativas ao ensino e à aprendizagem ainda não foram suficientemente equacionadas e queas implicações pedagógicas expressas no modelo de Bolonha, apesar de muito referidas, estão longe de ser consideradas, avaliadas ou implementadas. Podemos mesmo dizer que, 
em muitos casos, essa revisão curricular muito pressionada pelo tempo político não passou de uma operação de cosmética com objectivos econômicos associados e que está longe das mudanças profundas que se esperavam em várias dimensões. Sendo o ensino superior um contexto complexo e multidimensional, a nossa opção recaiu no estudo de uma das suas componentes que consideramos assumir particular relevância: a qualidade da aprendizagem dos estudantes compreendida a partir da sua própria perspectiva (perspectiva fenomenográfica).

A investigação sobre a aprendizagem dos estudantes no ensino superior inicia-se com Marton e Säljo (1976; 1976a) na Suécia a partir de estudos qualitativos. Parte de conceitos relacionados com a aprendizagem mecânica e a aprendizagem significativa propostos por Ausubel (1968) e Bloom (1972) e pelo modelo dos níveis de processamentosuperficiais(centrados em processos de simples memorização) e profundos(centrados em processos de compreensão) operacionalizados na mesma alturapor Craig e Lockart (1972). $\bigcirc$ resultado destes estudos estabelece uma estreita relação entre o processo de ensino, a avaliação e o tipo de abordagens à aprendizagem utilizadas pelos estudantes. Estudos posteriores (NEWBLE; JAEGER, 1983; THOMAS; BAIN, 1984) confirmam os resultados iniciais verificando que o comportamento dos alunos dependia, em grande medida, do tipo de avaliação prevista nos currículos acadêmicos e em particular da tipologia das provas de avaliação (mais direccionadas para reprodução de informação ou para processos compreensivos).

Nas últimas quatro décadas realizaram-se numerosos estudoscom base nos autorrelatos dos estudantesem que ressaltou uma forte relação entre as abordagens à aprendizagem e a percepção dos estudantes sobre o contexto em que esta decorria.A percepção do contexto de aprendizagem surge em estudos recentes como experiência no curso e reporta-se a aspectos relacionados com o bomensino, metas claras, carga de trabalho, avaliação adequada e competências genéricas (actualmente designadas de soft skills) desenvolvidas pelos estudantes no curso.

Um dos objectivos das pesquisas realizadas neste modelo (modelo SAL - Students Approaches to Learningl, inicialmente de cariz predominantemente qualitativo, consistia na elaboração de instrumentos que avaliassem os construtos identificados. As abordagens à aprendizagem apresentavam forte relevância para a compreensão da forma como os estudantes acediam ao 
Experiência no curso - contributo para a qualidade do ensino superior

conhecimento no ensino superior e para a definição de processos de aprendizagem de elevada qualidade (ENTWISTLE, 2009; CHALETA; ENTWISTLE, 20111 . A relação que esses estudos estabeleceram com a percepção dos estudantes sobre o seu contexto de aprendizagem levou à construção do CPQ - Questionário de Percepção do Curso (RAMSDEN; ENTWISTLE, 1981 ; ENTWISTLE; RAMSDEN, 1983) que se configurou como a primeira versão de um instrumento que se foi desenvolvendo ao longo do tempo e que, actualmente, adopta a denominação de CEQ - Questionário de Experiência do Curso (RAMSDEN, 1994; 2005; 2006). Das três versões existentes do instrumento (CEQ25, CEQ30 e CEQ36 em função do número de itens que integra cada versão) optamos pela adaptação do CEQ25 (CHALETA; SAMPAIO; SARAIVA; GRÁCIO; LEAL; SANTOS; SEBASTIÃO; CANDEIAS, 2012) por ser a mais utilizada recentemente em particular no Japão e na Austrália. Na Universidade de Sydney, os resultados do CEQ25têm implicações aonível do financiamento das faculdades, na avaliaçãodo desempenho (competitivo) em termos de ensino aonível do corpo docente em termos globais (nunca em termos de avaliação individual do desempenho do docente) podendo, inclusivamente, determinar mudanças nas directrizes das faculdades. Os resultados (divulgados 68 publicamente) podem determinar a reorganização do corpo docente em função da definição de grandes projectos estratégicos, a definição de políticas educativas alternativas e mudanças aonível das práticas tendo sempre como objectivo último melhorar a experiência do aluno no curso (GINNS; PROSSER; BARRIE, 2007). Recentemente, o CEQ25 tem sido utilizado como benchmark da Universidade de Sydney para comparar resultados de estudantes que frequentamas universidades de Queensland,Monash e Oxford no sentido de identificarem as melhores práticas ao níveldo ensino/aprendizagem. Mesmo as universidades que privilegiam a aprendizagem numa perspectiva de pesquisa intensiva estão a adoptar o instrumento com o objectivo final de melhorar a experiência do aluno na instituição dado que o CEQ25 se revelou como um indicador de desempenho apropriado para toda a gama de universidades (PROSSER; BARRIE, 2003).

O CEQ 25 integra 24 itens que se distribuem por cinco factores (Bom Ensino, Metas Claras, Avaliação Apropriada, Carga de Trabalho Apropriada e Competências Genéricas) e um item que avalia a satisfação com a qualidade do curso em geral (LEUNG; GINNS; KEMBER, 2008). 
Em relação ao bom ensino um dos aspectos mais relevantes diz respeito à experiência do professor que constitui um dos factores-chavepara a qualidade do ensino e da aprendizagem (MINTON, 1991).Para Ramsden (1994), não existem receitas certas nem técnicas infalíveis para o bom ensino e a eficácia educacional depende essencialmente do profissionalismo, da experiência e do compromisso dos agentes envolvidos no processo. Neste sentido, apresenta três áreas-chave para melhorar a qualidadedo processo de ensino e de aprendizagem nas instituições de ensino superior, designadamente: i) a experiência dos professores ii) a liderança acadêmica forte, motivada e de excelência; iii) os métodos de avaliação adequados à realidade envolvente.

Ramsden (1994) refere que os professores mais novos e menos experientes tentam adaptar-se ao contexto existente e, muitas vezes, consideram o ensino essencialmente como forma de transmissão eficientede conhecimento sem perceberem as implicações que essa concepção reprodutiva tem para a aprendizagemdos estudantes. Por seu turno, os professores especialistas e mais experientes consideram o ensino comouma forma de interagir com os alunos e monitorizar a sua aprendizagem. Essa visão também pode envolver alguma apresentação de informação mas a principal intenção é que, no ambiente educacional, seja explícito o que se espera dos alunos. Assim, focaliza a suaatenção nos acontecimentos que ocorrem na sala de aula compreendendo o valor da reflexão sobre a prática como uma forma de adaptação e melhoria.

Para que haja qualidade de aprendizagem nas instituições, é necessário reconhecer e premiar o bom ensino, proporcionar um bom ambiente e uma liderança de excelência de modo a que os professores se sintam capacitados para responder às necessidadesdos alunos. A liderança constitui-secomo uma variável crucial para determinar a satisfação e o sucesso, criando as condições para que os alunos aprendam num ambiente onde possam assumir a responsabilidade pela sua própria aprendizagem e uma envolvência de respeito e de cooperação (DISETH; PALLESEN; BRUNBORG; LARSEN, 2009).Fundamental, também, a criação de equipes de docentes que se norteiem por objectivos comuns e a monitorização contínua dos efeitos do ensino (RAMSDEN, 2006). É também fundamental estabelecer metas claras que permitam aos estudantes saber o que se espera deles e cargas de trabalho adequadas pois a investigação já provou que fortes cargas de trabalho determinam que os estudantes utilizem abordagens mais superficiais no seu processo de estudo e 
de aprendizagem (BLAND; RUFFIN, 1992; ENTWISTLE, 2009; RAMSDEN, 2004; 2006).

Um outro factor relevante diz respeito à avaliação da aprendizagem. Aavaliação fornece indicações sobre o tipo de aprendizagem e, consequentemente, sobre o ensino necessário para obter a qualidade desejada e percebida. Assim, o processo de avaliação do ensino, identificando os pontos fortes e os pontos fracos, bem como o processo de avaliação de cursos e das instituições, através de indicadores quantitativos de eficácia ltais como os resultados obtidos a partir do CEQ e outros instrumentos) podem contribuir para a melhoria contínua da qualidade do processo ensino e de aprendizagem pois permite a introdução de novas ideias, de melhores práticas e a remoção dos obstáculos que vão surgindo(RAMSDEN, 1994; 2006).

Na adaptação para a população portuguesa (CHALETA; SAMPAIO; SARAIVA; GRÁCIO; LEAL; SANTOS; SEBASTIÃO; CANDEIAS, 20 12 1), a solução final do instrumento que passamos a designar de CEQ-P ficou constituída por 23 itens e quatro factores como se pode ver na Figura 1.

Retirando o item que avalia a satisfação dos estudantes com o curso 70 que frequentam litem 25 no questionário originall), a estrutura testada de 22 itens agrupa-se em quatro factores: Bom Ensino (GT), com 8 itens, Competências Genéricas (GS), com 6 itens, Avaliação Apropriada (AA), com 4 itens e Carga de Trabalho Apropriada (AW) também com 4 itens. 
Figura 1

Representação Gráfica do Modelo Conceptual Teórico Final para CEQP

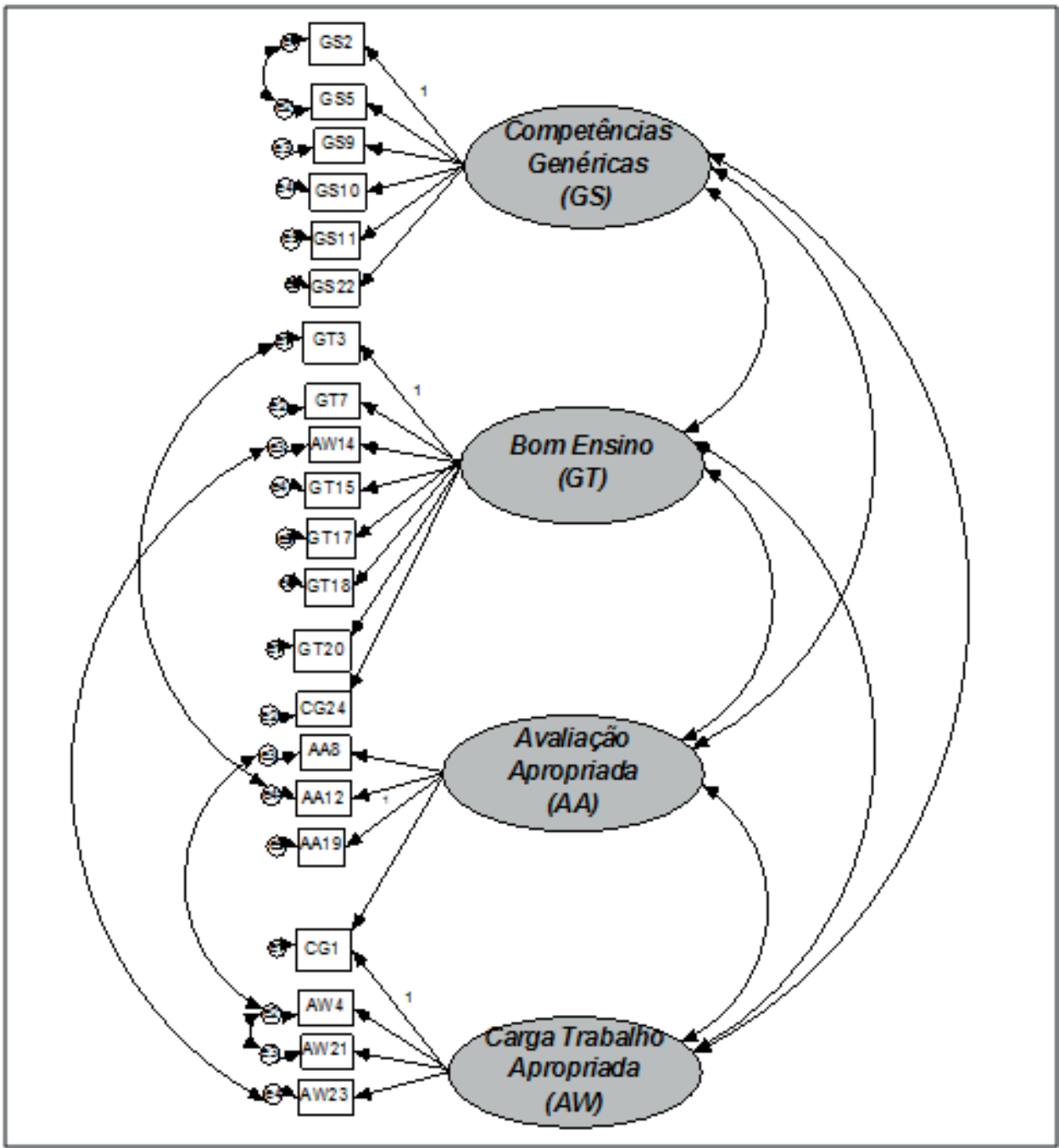

$([x 2(176)=593.50, p<0.001 ; A G F)=0.92 ; C F \mid=0.902 ;$

RMSEA=0.052; GFl=0.938]) 
$\bigcirc$ factor de Metas e Padrões Claros presente na estrutura original do instrumento não se identifica neste estudo, obtendo-se uma estrutura similar à encontrada por Fryer, Ginns, Wlaker e Nakao (201 1) na adaptação do instrumento para o contexto japonês.

\section{Método}

Quatro anos após a adaptação curricular dos cursos segundo as directivas de Bolonha ltodos os estudantes se encontram a frequentar os cursos neste modelo) e levando em conta a importância da percepção dos estudantes sobre o contexto educacional para a qualidade daaprendizagem, constituiu objectivo central deste estudo compreender como os estudantes que frequentam $\circ 1^{\circ}$ e o $3^{\circ}$ ano de cursos de Licenciatura de Ciências e Tecnologia e Ciências Sociais de três instituições de ensino superior portuguesaspercepcionam o curso que frequentam e qual o seu nível de satisfação nesses cursos.

De modo específico, pretendemos identificar diferenças i) entre estudantes que frequentam cursos de áreas científicas diferentes, i.e., Ciências

72 Sociais e Ciências e Tecnologia, ii) entre estudantes que frequentam diferentes cursos, iii) entre os estudantes que frequentam o $1^{\circ}$ ano e $\circ 3^{\circ}$ ano dos cursos, iv) entre os estudantes em função do gênero, v) entre os estudantes que frequentam diferentes instituições de ensino superior e, por fim vil verificar o nível de satisfação dos estudantes com a qualidade dos cursos que frequentam.

\section{Participantes}

instrumento foi aplicado a 872 estudantes $131.4 \%$ do sexo masculino e $68.6 \%$ do sexo feminino) que frequentavam cursos de Licenciatura de duas instituições de ensino superior públicas (Universidade de Évora e Universidade de Lisboa) e uma privada (ISPA - Instituto Universitário). 
Tabela 1

Distribuição dos alunos por escola/Curso

\begin{tabular}{|c|c|c|c|c|c|}
\hline Escola & Curso & $\mathrm{N}$ & $\%$ & $\mathrm{~N}$ & $\%$ \\
\hline \multirow{7}{*}{$\begin{array}{l}\text { Ciencias e } \\
\text { Tecnologias }\end{array}$} & Engenharia Civil & 43 & 4.9 & \multirow{7}{*}{295} & \multirow{7}{*}{33.8} \\
\hline & Biologia & 40 & 4.6 & & \\
\hline & Engenharia Informática & 29 & 3.3 & & \\
\hline & Medicina Veterinária & 45 & 5.2 & & \\
\hline & Arquitectura Paisagista & 42 & 4.8 & & \\
\hline & Reabilitação Psicomotora & 60 & 6.9 & & \\
\hline & Ciências do Desporto & 36 & 4.1 & & \\
\hline \multirow{8}{*}{ Ciências Sociais } & Relações Internacionais & 29 & 3.3 & \multirow{8}{*}{577} & \multirow{8}{*}{66.2} \\
\hline & Gestão & 50 & 5.7 & & \\
\hline & Economia & 41 & 4.7 & & \\
\hline & Educação Básica & 30 & 3.4 & & \\
\hline & Ciências da Educação & 21 & 2.4 & & \\
\hline & Sociologia & 41 & 4.7 & & \\
\hline & Psicologia & 341 & 39.1 & & \\
\hline & $\begin{array}{l}\text { Ciências da Informação e } \\
\text { da Documentação }\end{array}$ & 26 & 2.8 & & \\
\hline & \multicolumn{3}{|l|}{ Total } & 872 & 100 \\
\hline
\end{tabular}

Desses, alunos 33.8\% frequentam cursos da área de Ciências e Tecnologia e $66.2 \%$ cursos da área de Ciências Sociais. Em relação ao ano de frequência, $64.6 \%$ frequentam o $1^{\circ}$ ano e $35.4 \%$ frequentam o $3^{\circ}$ ano dos cursos. A idade dos sujeitos oscilava entre 17 e 62 anos, situando-se a média em 22 anos (desvio-padrão de 7.19 ).

2. Instrumento e procedimentos

O instrumento a que recorremos neste estudo, CEQ-P - Questionário de Experiência no Cursoresulta da adaptação do CEQ25 (RAMSDEN, 2005) para a população portuguesa por CHALETA; SAMPAIO; SARAIVA; GRÁCIO; 
Experiência no curso - contributo para a qualidade do ensino superior

LEAL; SANTOS; SEBASTIÃO; CANDEIAS (2012) com uma solução final de 23 itens em que 22 dão corpo à estrutura factorial e, um último, tratado de forma independente, avalia a satisfação dos estudantes com a qualidade do curso.

Os 4 factores (escalas) obtidos consistem em:

1 - Bom ensino, escala caracterizada por práticas pedagógicas que incluem fornecer feedback útil e oportuno, explicações claras, motivar os alunos, tornar o curso interessante e atenção a problemas de compreensão dos alunos. As pontuações mais baixas nessa escala estão associadas com a percepção dos alunos de que tais práticas ocorrem com menos frequência (itens $3,7,15,17,181$.

2 - Avaliação adequada, escala paraa avaliação de ordem superior que considera o pensar e o compreender ao invés da recordação factual simples. Essa escala não investiga outros aspectos importantes das práticas de avaliação, tais como a congruência da avaliação com o material realmente ensinado, o nível de dificuldade e a consistência da qualidade da avaliação (itens 8, 12, 19).

3 - Carga de trabalho adequada, escala relativa à percepção de 74 cargas de trabalho razoáveis (itens 1, 4, 21, 23), sendo que os itens com valores mais elevados indicam nessa escala adequabilidade da carga de trabalho. Cargas de trabalho muito pesadas tendem a impedir os estudantes de participarem e compreenderem o material que estão a aprender. Muitos estudantes adoptam abordagens superficiais à aprendizagem como uma estratégia para lidar com cargas de trabalho elevadas.

4 - Competências gerais, escala cujos itens reflectem a medida em que os alunos percebem que os seus estudos têm favorecido o desenvolvimento de competências genéricas reconhecidas pela universidade como sendo um resultado valioso do ensino universitário, além de disciplinar as habilidades e conhecimentos específicos (itens 2, 5, 9, 10, 11, 22).

Para o tratamento estatístico dos dados recorremos ao SPSS 21 (Statistical Package for Social Sciences). 
Resultados

Os resultados que se apresentam respondem aos objectivos definidos anteriormente em termos da experiência dos estudantes no curso e do seu nível de satisfação.

Pela análise da Tabela 2 podemos observar que existem diferenças estatisticamente significativas entre os estudantes que frequentam cursos de Ciências Sociais e de Ciências e Tecnologia aonível do Bom Ensino (média mais elevada nos estudantes que frequentam cursos de Ciências Sociais), Avaliação Adequada e Carga de Trabalho (médias mais elevada nos estudantes que frequentam cursos de Ciências e Tecnologia).

\section{Tabela 2}

Experiência no curso por relação com as áreas científicas/ECT; ECS

\begin{tabular}{|l|c|c|c|c|c|}
\hline \multirow{2}{*}{ Escalas } & \multicolumn{2}{|c|}{ CT } & \multicolumn{2}{c|}{ CS } & \multirow{2}{*}{$\mathbf{p}$} \\
\cline { 2 - 5 } & M & DP & M & DP & \\
\hline Bom Ensino (BE) & 2.99 & .630 & 3,26 & .621 & .000 \\
\hline Avaliação Adequada (AA) & 2.90 & .644 & 2,64 & .583 & .000 \\
\hline Carga de Trabalho (CT) & 3.25 & .462 & 3,05 & .538 & .000 \\
\hline $\begin{array}{l}\text { Competências Genéricas } \\
\text { (CG) }\end{array}$ & 3.67 & .523 & 3,69 & .549 & .474 \\
\hline
\end{tabular}

Os resultados obtidos em relação à experiência dos estudantes nos respectivos cursos indicam diferenças estatisticamente significativas ( $p=.000$ ) em todas as escalas (Tabela 3). Verifica-se que, na escala de Bom Ensino, quatro dos sete cursos de Ciências e Tecnologia se encontram abaixo do ponto médio (3) apesar de incluir o curso de Ciências do Desporto o que obtém média mais elevada $(M=3.46)$ quando considerada a globalidade dos cursos. Em relação a essa escala, nos cursos de Ciências Sociais, Psicologia é o curso que apresenta média mais elevada ( $M=3.37)$. Seguem-se os cursos de Ciências da Informação e Documentação $(M=3.27)$ e Gestão $(M=3.26)$. A média mais baixa, situada abaixo do ponto médio (3), nessa área científica, encontra-se no curso de Educação Básica $(M=2.82)$.

Avaliação Adequada encontra-se acima da média apenas em cursos de Ciências e Tecnologia (Biologia e Medicina Veterinária). No que se 
Experiência no curso - contributo para a qualidade do ensino superior

refere à Carga de Trabalho, encontramos as médias mais elevadas nos cursos de Medicina Veterinária $(M=3.41)$ e Ciências do Desporto $(M=3.33)$ e Educação Básica (M=3.32) e menores nos cursos de Economia $(M=2.95)$ e Psicologia (M=2.99) o que indica cargas de trabalho consideradas pesadas nesses cursos.

Tabela 3

Experiência dos estudantes no curso

\begin{tabular}{|c|c|c|c|c|c|c|c|c|c|}
\hline \multirow{2}{*}{ Escola } & \multirow{2}{*}{ Curso } & \multicolumn{2}{|c|}{$\mathrm{BE}^{* *}$} & \multicolumn{2}{|c|}{$\mathrm{AA}^{* *}$} & \multicolumn{2}{|c|}{$\mathrm{CT}^{* *}$} & \multicolumn{2}{|c|}{ CG** } \\
\hline & & $M$ & DP & $M$ & $\mathrm{DP}$ & $M$ & $\mathrm{DP}$ & $M$ & DP \\
\hline \multirow{7}{*}{ ECT } & $\begin{array}{l}\text { Engenharia } \\
\text { Civil }\end{array}$ & 2.90 & .528 & 2.91 & .494 & 3.19 & .438 & 3.41 & .429 \\
\hline & Biologia & 2.67 & .708 & 3.11 & .567 & 3.17 & .520 & 3.43 & .582 \\
\hline & $\begin{array}{l}\text { Engenharia } \\
\text { Informática }\end{array}$ & 2.97 & .585 & 2.66 & .804 & 3.27 & .463 & 3.30 & .528 \\
\hline & $\begin{array}{l}\text { Medicina } \\
\text { Veterinária }\end{array}$ & 2.60 & .542 & 3.10 & .681 & 3.41 & .471 & 3.75 & .542 \\
\hline & $\begin{array}{l}\text { Arquitectura } \\
\text { Paisagista }\end{array}$ & 3.12 & .555 & 2.75 & .579 & 3.26 & .455 & 3.81 & .437 \\
\hline & $\begin{array}{l}\text { Reabilitação } \\
\text { Psicomotora }\end{array}$ & 3.18 & .610 & 2.79 & .619 & 3.15 & .439 & 3.91 & .389 \\
\hline & $\begin{array}{l}\text { Ciências do } \\
\text { Desporto }\end{array}$ & 3.46 & .416 & 2.94 & .701 & 3.33 & .422 & 3.87 & .438 \\
\hline \multirow{8}{*}{ ECS } & $\begin{array}{l}\text { Relações Inter- } \\
\text { nacionais }\end{array}$ & 3.15 & .526 & 2.69 & .577 & 3.01 & .519 & 3.60 & .529 \\
\hline & Gestão & 3.26 & .662 & 2.73 & .570 & 3.21 & .484 & 3.76 & .449 \\
\hline & Economia & 3.07 & .552 & 2.58 & .646 & 2.95 & .532 & 3.78 & .573 \\
\hline & $\begin{array}{l}\text { Educação } \\
\text { Básica }\end{array}$ & 2.82 & .737 & 2.94 & .554 & 3.32 & .553 & 3.73 & .652 \\
\hline & $\begin{array}{l}\text { Ciências da } \\
\text { Educação }\end{array}$ & 3.06 & .689 & 2.90 & .700 & 3.01 & .490 & 3.50 & .579 \\
\hline & Sociologia & 3.04 & .786 & 2.61 & .673 & 3.15 & .599 & 3.74 & .685 \\
\hline & Psicologia & 3.37 & .560 & 2.57 & .535 & 2.99 & .529 & 3.68 & .529 \\
\hline & $\begin{array}{l}\text { Ciências } \\
\text { Informação e } \\
\text { Documenta- } \\
\text { ção }\end{array}$ & 3.27 & .643 & 2.99 & .663 & 3.22 & .558 & 3.65 & .579 \\
\hline
\end{tabular}


A análise dos resultados por relação com o ano de frequência $\left(1^{\circ}\right.$ ano e $3^{\circ}$ anolindica diferenças estatisticamente significativas nas escalas de Carga de Trabalho ( $p=000$ ) e Competências Genéricas ( $p=000)$ sendo as médias mais elevadas encontradas nos estudantes de $3^{\circ}$ ano (Tabela 4).

\section{Tabela 4}

Experiência no curso por relação com o ano de frequência $/ 1^{\circ}$ ano; $3^{\circ}$ ano

\begin{tabular}{|l|c|c|c|c|c|}
\hline \multirow{2}{*}{\multicolumn{1}{|c|}{ Escolas }} & \multicolumn{2}{|c|}{$\mathbf{1}^{\mathbf{0}}$ Ano } & \multicolumn{2}{c|}{$\mathbf{3}^{\circ}$ Ano } & \multirow{2}{*}{$\mathbf{p}$} \\
\cline { 2 - 6 } & M & DP & M & DP & \\
\hline Bom Ensino (BE) & 3.19 & .627 & 3.19 & .656 & .192 \\
\hline Avaliação Adequada (AA) & 2.69 & .600 & 2.79 & .640 & .024 \\
\hline Carga de Trabalho (CT) & 3.07 & .522 & 3.21 & .509 & .000 \\
\hline Competências Genéricas (CG) & 3.61 & .521 & 3.81 & .552 & .000 \\
\hline
\end{tabular}

No que se refere às diferenças de gênero, como podemos ver na Tabela 5, apenas se encontram diferenças estatisticamente significativas em Competências Genéricas ( $p=000$ ) sendo que os valores mais elevados se encontram nas alunas.

Tabela 5

Experiência no curso por relação com o género

\begin{tabular}{|c|c|c|c|c|c|}
\hline \multirow{2}{*}{ Escolas } & \multicolumn{2}{|c|}{ Masculino } & \multicolumn{2}{c|}{ Feminino } & \multirow{2}{*}{ p } \\
\cline { 2 - 5 } & M & DP & M & DP & \\
\hline Bom Ensino (BE) & 3.08 & .637 & 3.21 & .634 & .008 \\
\hline Avaliação Adequada (AA) & 2.78 & .667 & 2.71 & .590 & .100 \\
\hline Carga de Trabalho (CT) & 3.17 & .528 & 3.09 & .518 & .060 \\
\hline Competências Genéricas (CG) & 3.55 & .577 & 3.74 & .511 & .000 \\
\hline
\end{tabular}

A análise em função da instituição permite-nos observar diferenças estatisticamente significativas em todas as escalas excepto Competências Genéricas. Com relação ao do Bom Ensino, encontramos nas instituições valores acima da média sendo a média mais elevada no ISPA. Em relação à Avaliação Adequada, os valores encontram-se abaixo da média em todas 
as instituições sendo a mais baixa a da Universidade de Lisboa ( $M=2.50)$. Carga de Trabalho adequada obtém a média mais elevada na Universidade de Évora $(M=3.19)$.

Tabela 6

Experiência no curso por relação com a instituição

\begin{tabular}{|c|c|c|c|c|c|c|c|}
\hline \multirow{2}{*}{ Escalas } & \multicolumn{2}{|c|}{ U. Évora } & \multicolumn{2}{|c|}{ U. Lisboa } & \multicolumn{2}{c|}{ ISPA } & \multirow{2}{*}{ p } \\
\cline { 2 - 7 } & M & DP & M & DP & M & DP & \\
\hline Bom Ensino (BE) & 3.04 & .650 & 3.16 & .556 & 3.49 & .529 & .000 \\
\hline Avaliação Adequada (AA) & 2.83 & .642 & 2.50 & .528 & 2.61 & .536 & .000 \\
\hline Carga de Trabalho (CT) & 3.19 & .503 & 2.93 & .527 & 3.05 & .528 & .000 \\
\hline $\begin{array}{c}\text { Competências Genéricas } \\
\text { (CG) }\end{array}$ & 3.68 & .547 & 3.59 & .503 & 3.74 & .536 & .044 \\
\hline
\end{tabular}

Em relação à satisfação dos estudantes com a qualidade dos cursos que frequentam, podemos observar (Tabela 7) queem três dos cursos (Engenharia Civil, Medicina Veterinária e Educação Básica) menos da metade

78 dos estudantes refere satisfação com a qualidade dos cursos. Em dois dos cursos (Ciências do Desporto e Relações Internacionais) não se encontra qualquer referência dos estudantes reveladora de insatisfação. 
Tabela 7

Satisfação com a qualidade do curso

\begin{tabular}{|c|c|c|c|c|c|}
\hline \multirow{4}{*}{$\begin{array}{c}\text { Cursos } \\
\text { Engenharia Civil }\end{array}$} & \multicolumn{5}{|c|}{ Satisfação (\%) } \\
\hline & \multicolumn{5}{|c|}{1 (insatisfação) -- $3-$ (satisfação) 5} \\
\hline & 2.3 & 9.3 & \multirow{2}{*}{44.2} & 41.9 & 2.3 \\
\hline & \multicolumn{2}{|c|}{11.6} & & \multicolumn{2}{|c|}{44.2} \\
\hline \multirow{2}{*}{ Biologia } & 2.5 & 12.5 & \multirow{2}{*}{25.0} & 55.0 & 5.0 \\
\hline & \multicolumn{2}{|c|}{15.0} & & \multicolumn{2}{|c|}{60.0} \\
\hline \multirow{2}{*}{ Engenharia Informática } & 0 & 13.8 & \multirow{2}{*}{31.0} & 44.8 & 10.3 \\
\hline & \multicolumn{2}{|c|}{13.8} & & \multicolumn{2}{|c|}{55.1} \\
\hline \multirow{2}{*}{ Medicina Veterinária } & 8.9 & 13.3 & \multirow{2}{*}{31.1} & 42.2 & 4.4 \\
\hline & \multicolumn{2}{|c|}{22.2} & & \multicolumn{2}{|c|}{46.6} \\
\hline \multirow{2}{*}{ Arquitectura Paisagista } & 2.4 & 4.8 & \multirow{2}{*}{21.4} & 57.1 & 14.3 \\
\hline & \multicolumn{2}{|c|}{7.2} & & \multicolumn{2}{|c|}{71.4} \\
\hline \multirow{2}{*}{ Reabilitação Psicomotora } & 0 & 3.3 & \multirow{2}{*}{10.0} & 68.3 & 18.3 \\
\hline & \multicolumn{2}{|c|}{3.3} & & \multicolumn{2}{|c|}{86.6} \\
\hline \multirow{2}{*}{ Ciências do Desporto } & 0 & 0 & \multirow{2}{*}{13.9} & 69.4 & 16.7 \\
\hline & C & & & \multicolumn{2}{|c|}{87.7} \\
\hline \multirow{2}{*}{ Relações Internacionais } & 0 & 0 & \multirow{2}{*}{17.2} & 55.2 & 27.6 \\
\hline & C & & & & \\
\hline \multirow{2}{*}{ Gestão } & 0 & 4.0 & $2 \cap \cap$ & 64.0 & 12.0 \\
\hline & 4 & & 20.0 & & \\
\hline 50 & 2.4 & 7.3 & 10 & 61.0 & 9.8 \\
\hline Economia & 9 & & 19.5 & & \\
\hline Fdurarãn Rácirg & 13.3 & 23.3 & 200 & 43.0 & 0 \\
\hline Equcaçao Basıca & 36 & & 20.0 & & \\
\hline 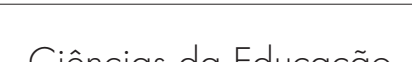 & 9.5 & 4.8 & $?$ & 52.4 & 4.8 \\
\hline Ciencias da Educaçao & 14 & & 28.0 & & \\
\hline$c_{0}$ & 4.9 & 17.1 & 260 & 31.7 & 19.5 \\
\hline SOClologia & 22 & & 20.0 & & \\
\hline
\end{tabular}


Experiência no curso - contributo para a qualidade do ensino superior

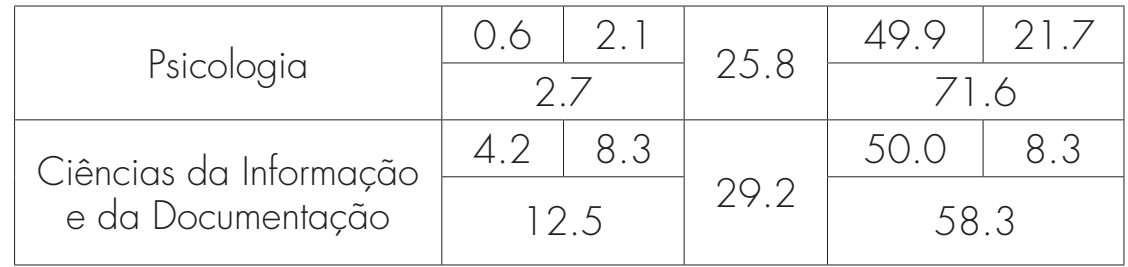

A análise dos dados encontra diferenças estatisticamente significativas entre os cursos $(p=.000)$ sendo os estudantes que referem maior satisfação lacima de $80 \%$ ) os que frequentam os cursos de Ciências do Desporto, Reabilitação Psicomotora e Relações Internacionais.

Na Tabela 8, podemos observar que a maioria dos estudantes das três instituições (Universidade de Évora, Universidade de Lisboa e ISPA Lisboa) está satisfeita com a qualidade dos cursos que frequentam.

\section{Tabela 8}

Satisfação dos estudantes com a qualidade do curso/Instituições

\begin{tabular}{|c|c|c|c|c|c|}
\hline \multirow{2}{*}{ Instituições } & \multicolumn{5}{|c|}{ Satisfação (\%) } \\
\hline & \multicolumn{5}{|c|}{1 (insatisfação) - $3-$ - (satisfação) 5} \\
\hline \multirow{2}{*}{ Universidade de Évora } & 2.4 & 7.8 & \multirow{2}{*}{24.4} & 54.9 & 10.5 \\
\hline & \multicolumn{2}{|c|}{10.2} & & \multicolumn{2}{|c|}{65.4} \\
\hline \multirow{2}{*}{ Universidade de Lisboa } & 2.1 & 5.0 & \multirow{2}{*}{24.4} & 50.6 & 17.9 \\
\hline & \multicolumn{2}{|c|}{7.1} & & \multicolumn{2}{|c|}{68.5} \\
\hline \multirow{2}{*}{ ISPA Lisboa } & 0 & 0.9 & \multirow{2}{*}{24.8} & 50.5 & 23.9 \\
\hline & & & & \multicolumn{2}{|c|}{74.4} \\
\hline
\end{tabular}

A análise estatística realizada indica diferenças significativas entre as instituições ( $p=.000$ ) sendo o ISPA Lisboa a que apresenta estudantes mais satisfeitos com a qualidade do curso. 


\section{Tabela 9}

Satisfação dos estudantes com o curso que frequentam/ área científica

\begin{tabular}{|c|c|c|c|c|c|}
\hline \multirow{2}{*}{ Áreas Científicas } & \multicolumn{5}{|c|}{ Satisfação (\%) } \\
\hline & \multicolumn{5}{|c|}{1 (insatisfação) -3-3- (satisfação) 5} \\
\hline \multirow{2}{*}{ Ciências e Tecnologia } & 2. & 7.8 & \multirow{2}{*}{24.4} & 54.9 & 10.5 \\
\hline & \multicolumn{2}{|c|}{10.2} & & \multicolumn{2}{|c|}{65.4} \\
\hline \multirow{2}{*}{ Ciências Sociais } & 2. & 5.0 & \multirow{2}{*}{24.4} & 50.6 & 17.9 \\
\hline & & & & \multicolumn{2}{|c|}{8.5} \\
\hline
\end{tabular}

Em relação às áreas científicas, os valores obtidos são muito semelhantes não se encontrando diferenças estatisticamente significativas ao nível da satisfação entre os estudantes que frequentam cursos dessas áreas científicas.

Tabela 10

Satisfação dos estudantes com o curso que frequentam $/ 1^{\circ}$ ano; $3^{\circ}$ ano

\begin{tabular}{|c|c|c|c|c|}
\hline \multirow{2}{*}{ Ano } & \multicolumn{4}{|c|}{ Satisfação (\%) } \\
\hline & \multicolumn{4}{|c|}{1 (insatisfação) -3-- (satisfação) 5} \\
\hline \multirow{2}{*}{$1^{\circ}$ Ano } & 1.4 & \multirow{2}{*}{27.2} & 51.5 & 15.88 \\
\hline & 5.4 & & \multicolumn{2}{|c|}{67.3} \\
\hline \multirow{2}{*}{$3^{\circ}$ Ano } & 3.6 & \multirow{2}{*}{19.4} & \multirow{2}{*}{\multicolumn{2}{|c|}{$\begin{array}{c}53.114 .6 \\
\mathbf{6 7 . 7}\end{array}$}} \\
\hline & 13.0 & & & \\
\hline
\end{tabular}

A Tabela 10 permite-nos verificar níveis de satisfação idênticos entre estudantes de $1^{\circ}$ e $3^{\circ}$ ano (acima de $60 \%$ ), no entanto verifica-se que mais estudantes do $3^{\circ}$ ano referem insatisfação com a qualidade do curso o que se traduz no resultado obtido pela análise estatística que, nesse caso, revela diferenças estatisticamente significativas $(p=001)$.

A análise dos dados, tendo em conta o sexo dos estudantes, indica maior satisfação com a qualidade do curso por parte das jovens estudantes. 
Experiência no curso - contributo para a qualidade do ensino superior

Tabela 11

Satisfação dos estudantes com o curso que frequentam / sexo

\begin{tabular}{|c|c|c|c|c|c|}
\hline \multirow{2}{*}{ Sexo } & \multicolumn{5}{|c|}{ Satisfação (\%) } \\
\hline & \multicolumn{5}{|c|}{1 (insatisfação) -3-3-(satisfação) 5} \\
\hline \multirow{2}{*}{ Masculino } & 1.8 & 9.2 & \multirow{2}{*}{30.0} & 48.4 & 10.6 \\
\hline & & & & \multicolumn{2}{|c|}{50.0} \\
\hline \multirow{2}{*}{ Feminino } & 2.3 & 4.5 & \multirow{2}{*}{21.9} & 53.8 & 17.5 \\
\hline & & & & \multicolumn{2}{|c|}{71.3} \\
\hline
\end{tabular}

A análise estatística realizada indica diferenças significativas entre os $\operatorname{sexos}(p=001)$.

\section{Conclusões}

Os resultados obtidos em termos de área científica indicam, neste estudo, maior evidência de bom ensino nos cursos de Ciências Sociais e avalia-
ção e carga de trabalho mais adequadas nos cursos de Ciências e Tecnologia.

Os resultados obtidos em relação à experiência dos estudantes nos respectivos cursos indicam diferenças significativas em todas as escalas. Verifica-se que, na escala de Bom Ensino, quatro dos sete cursos de Ciências e Tecnologia se encontram abaixo do ponto médio (3) quando considerada a globalidade dos cursos. Em dois desses cursos Avaliação Apropriada obtém valores abaixo da média (Engenharia Civil e Engenharia Informática) e dois obtêm os valores mais elevados da escala por relação com a globalidade dos cursos. Carga de Trabalho, nesses quatro cursos, obtém valores acima do ponto médio pelo que será necessário analisar com maior detalhe as causas que determinam os resultados ao nível do Bom Ensino e da Avaliação Apropriada tendo em conta os aspectos referidos por Minton (1991). Por outro lado, é de fazer notar que, com excepção dos dois cursos de Engenharia (Civil e Informática), todos os demais cursos apresentam valores abaixo da média para Avaliação Apropriada pelo que é necessário analisar com detalhe os aspectos que os estudantes percepcionam como menos adequados num contexto em querevelam maioritariamente Bom Ensino e Carga de Trabalho 
Adequada tendo por referência a literatura sobre o assunto (BLAND e RUFFIN, 1992; ENTWISTLE, 2009; RAMSDEN, 1994; 2006).

No que se refere ao desenvolvimento de Competências Genéricas, em todos os cursos a percepção é de que elas são desenvolvidas.

A análise dos resultados por relação com o ano de frequência indica uma apreciação mais favorável dos estudantes de $3^{\circ}$ ano nas escalas de Carga de Trabalho e Competências Genéricas. No que se refere ao gênero, são as estudantes as que mais valorizam o desenvolvimento de Competências Genéricas nos cursos que frequentam.

A análise em função das instituições permite-nos observar diferenças estatisticamente significativas em todas as escalas excepto Competência Genéricas. Em Bom Ensino, encontramos nas instituições valores acima da média sendo a média mais elevada no ISPA. Em relação à Avaliação Adequada, os valores encontram-se abaixo da média em todas as instituições sendo a mais baixa a da Universidade de Lisboa. Carga de Trabalho é percebida como mais adequada na Universidade de Évora.

Quanto à satisfação dos estudantes com a qualidade dos cursos que frequentam, podemos observar que a maioria dos estudantes das três instituições (Universidade de Évora, Universidade de Lisboa e ISPA Lisboa) está satisfeita com a qualidade dos cursos que frequentam sendo o ISPA Lisboa a que apresenta valores mais elevados de satisfação.

Na análise das científicas não se encontram diferenças estatisticamente significativas aonível da satisfação. Em relação aos cursos apenas três de entre os quinze analisados revelam menor satisfação, embora os que manifestam insatisfação (com excepção de Educação Básica) não ultrapassem um quarto dos alunos respondentes.

Quando analisadas as respostas tendo em conta $\circ 1^{\circ}$ e o $3^{\circ}$ ano, verificamos que de modo geral existe satisfação em ambos os grupos e que a maior insatisfação surge nos estudantes de $3^{\circ}$ ano embora os valores correspondentes à insatisfação sejam bastante baixos. A satisfação por relação com o gênero indica claramente maior satisfação do sexo feminino. 


\section{Referências}

AAUSUBEL, David. Educational psychology: a cognitive view. New York and Toronto: Holt, Rinehart and Winston, 1968.

BLAND, Carol; RUFFIN, Mack. Characteristics of a productive research environment: Literature review. Academic Medicine, n. 67, p. 385-397, 1992.

$\mathrm{BLOOM}$, Benjamin. Taxonomy of educational objectives - the classification of educational goals. Handbook I: cognitive domain. New York: McKay, 1972.

CHALETA, Elisa; ENTWISTLE, Noel. Abordagens à aprendizagem em estudantes o ensino superior português - revisão do ASSIST - sv. Educação: Temas e Problemas, 2011 . (Numero especial sobre o Ensino Superior).

CHALETA, Elisa;SAMPAIO, Ana;SARAIVA, Margarida;GRÁCIO, Luísa;LEAL, Fátima;SANTOS, Graça;SEBASTIÃO, Luís; CANDEIAS, Adelinda. Adaptação e validação do CEQ Questionário de Experiência no Curso - Contributo para a qualidade do ensino superior português. TMQ - Techniques, Methodologies and Quality, n. 3,p. 187-204,2012.

CRAIG, Fergus; LOCKHART, Robert. Levels of processing: a framework for memory research. 84 Journal of Verbal Learning and Verbal Behavior, n. 1 1, p. 671-684, 1972.

DISETH, Anna;PALLESEN, Soren; BRUNBORG, Geir; LARSEN, Soren. Academic achievement among first semester undergraduate psychology students: the role of course experience, effort, motives and learning strategies. Higher Education, n. 59, p. 335-352, 2009.

ENTWISTLE, Noel. Teaching for understanding at university. Deep Approaches and distinctive Ways of Thinking. United Kingdon: Palgrave and Macmillan, 2009.

FRYER, Luke;GINNS, Paul;WALKER, Richard;NAKAO, Keisuke. The adaptation and validation of CEQ and the R-SPQ-2F to the Japanese tertiary environment. British Journal of Educational Psychology, p. 1-15, 2011.

GINNS, Paul;PROSSER, Michael;BARRIE, Simon. Students' perceptions of teaching quality in higher education: The perspective of currently enrolled students. Studies in Higher Education, n. 32, p. 603-615, 2007.

LEUNG, Doris; GINNS, Paul; KEMBER, David. Examining the cultural specificity of approaches to learning in universities in Hong Kong and Sydney. Journal of Cross-Cultural Psychology, n. 39, v. 3, p. $251-266,2008$. 
MARTON, Ference; SÄLÖ, Roger. On qualitative differences in learning: I - Outcome and Processes. British Journal of Educational Psychology, n. 46, p. 4-1 1, 1976.

MARTON, Ference; SÄLJÖ, Roger. On qualitative differences in learning: II - outcome as a function of de learner's conception of the task. British Journal of Educational Psychology, n.46, p. $115-127,1976 a$.

MINTON, David. Teaching skills in further and adult education. Basingstoke: Macmillan, 1991.

NEWBLE, Di; JAEGER, Kerry. The effect of assessments and examinations on the learning of medical students. Medical Education, n. 17, p. 3,p. 165-171, 1983.

PROSSER, Michael; BARRIE, Simon. Using a studentfocused learning perspective to align academic development with institutional quality assurance. Towards strategic staffdevelopment in higher education. Philadelphia: Blackwell, 2003.

RAMSDEN, Paul. Using research on student learning to enhance educational quality. Improving student learning -theory and practice. Oxford: Oxford Centre for Staff Development, 1994.

RAMSDEN, Paul. The course experience questionnaire.2005. Disponível em:<www.engsc. ac.uk/.../experiencequestionnaire >. Acesso em: 5 mar. 2011.

RAMSDEN, Paul. A performance indicator of teaching quality in higher education: the course experience questionnaire. Studies in Higher Education, n. 16, v. 2, p. 129-150, 2006.

THOMAS, P.; Goranson; BAIN, John. Contextual differences of learning approaches: The effects of assessments. Human Learning, n. 3, p. 222-240, 1984.

Profa. PhD Maria Elisa Chaleta Universidade de Évora | Portugal Departamento de Psicologia Centro de Investigação | CIEP-UÉ E-mail |mec@uevora.pt

Recebido 30 jun. 2014 Aceito 19 jul. 2014 